Universitas, Volumen 1, Número 2, 2008,19-26

๑) 2008 UNAN-León, Editorial Universitaria

\title{
Un Experimento sencillo para evaluar la Incertidumbre siguiendo la guia GUM ISO 1995 y utilizando el cálculo simbólico MAPLE 11.0
}

\author{
Gustavo Delgado ${ }^{1 *}$ y Bertram Nagel ${ }^{2}$
}

${ }^{1}$ Facultad de Ciencias y Tecnología, Departamento de Química, Laboratorio de Análisis de Trazas de Metales Pesados (LATMP), Edificio de Ciencias Básicas, León, Nicaragua. Tel: 505311 5013, ext: 1132. Fax: 5053114012. Universidad Nacional Autónoma de Nicaragua, León (UNAN-LEÓN).

${ }^{2} E Q L$, Consultor PTB (Instituto Alemán de Metrología), Leipzig, Alemania. Nagel08@aol.com

\section{RESUMEN}

El objetivo del presente estudio, es estimar la incertidumbre en la medición del área total de un triángulo aplicando la guía de estimación de la incertidumbre GUM ISO 1995 y utilizando el programa de cálculo simbólico MAPLE 11. La medición la realizó un grupo de 10 estudiantes de la maestría en Química y Gestión de la Calidad, utilizando el mismo objeto de medición y el mismo instrumento de medición. Se derivó el modelo matemático, se identificaron los componentes de las incertidumbres a través del diagrama de causa-efecto, se calcularon los coeficientes de sensibilidad utilizando el programa de cálculo simbólico Maple 11, se elaboró un balance de las incertidumbres y un diagrama de Pareto donde se reflejan las variables que más influyen en la incertidumbre del mensurando. Se calcularon las incertidumbres combinada y expandida con un factor de cobertura igual a t al 95\% de nivel de confianza y 22 grados de libertad efectivos. Finalmente, se evaluó el intervalo de confianza, encontrándose un valor de $(50.72 \pm 0.39) \mathrm{cm}^{2}$. La incertidumbre expandida relativa fue menor del $1 \%$, indicando buena precisión en el proceso de medición.

Palabras claves: Incertidumbre en la medición del área de un triángulo; Cálculo de coeficiente de sensibilidad utilizando Maple; Incertidumbre expandida relativa $\left(u_{E R}\right)$; Metrología química; Balance o presupuesto de las incertidumbres.

\section{INTRODUCCIÓN}

La armonización de todas las unidades al Sistema Internacional de unidades SI, es de vital importancia en los tratados comerciales. Esta ha sido una de las principales barreras técnicas que obstaculiza el comercio internacional. La incongruencia de las unidades ha provocado grandes pérdidas para un país y ganancias para otros. Inclusive, en una misma nación, si las incongruencias son graves pueden ser causa de procesos judiciales. Por eso, actualmente en los tratados comerciales los países pobres están implementando leyes nacionales para aplicar el uso del SI basado en el sistema métrico decimal y en sus unidades básicas, derivadas y suplementarias ${ }^{[1]}$.

Así por ejemplo, en la exportación de productos de valor comercial es necesario el cumplimiento con especificaciones del país comprador basado en el sistema SI. De esta manera, el producto va acompañado con un informe de la conformidad ${ }^{[2,3]}$ extendido por los organismos competentes (organismos evaluador de la conformidad, laboratorios de ensayos acreditados), especificando el valor del parámetro requerido o mensurando con su incertidumbre a un nivel de confiabilidad establecida ${ }^{[4]}$. Los compradores disponen de una guía donde se describe el método que tienen que cumplir los laboratorios de ensayos o de calibración para declarar los resultados y la evaluación del cumplimiento con la especificación del producto dentro de los límites establecidos ${ }^{[5]}$ en los acuerdos comerciales. Estos límites, requieren que cada laboratorio de ensayo disponga de un procedimiento documentado para evaluar la incertidumbre ${ }^{[-9]}$ en los procesos de medición del parámetro o mensurando que indica la calidad del producto.

Dentro de este contexto, la metrología química desempeña un papel muy importante [10,11] y el personal técnico de un laboratorio de ensayo o de calibración deberá tener la competencia para evaluar el mensurando y estimar su incertidumbre asociada ${ }^{[4,5]}$. Con el fin de introducir a los estudiantes de ciencias químicas y físicas en el tema de la metrología, se ha reportado el análisis de la incertidumbre en los cálculos del área y el perímetro de un rectángulo utilizando el análisis estadístico clásico y el cálculo del error máximo probable ${ }^{[12]}$. En el presente estudio, se pretende evaluar el área de un triángulo y estimar su incertidumbre siguiendo la guía GUM ISO $1995^{[6]}$ y utilizando el programa de cálculo simbólico MAPLE[13].

Las mediciones la realizaron un grupo de 10 estudiantes de la maestría en Química y Gestión de la Calidad, sobre la misma figura geométrica y utilizando el mismo instrumento de medición, una regla graduada en centimetros.

\footnotetext{
*Autor para correspondencia: gusdel53@yahoo.es
} 


\section{TEORÍA}

\section{Ley de propagación de la incertidumbre}

Un proceso de medición puede representarse de acuerdo al "enfoque de proceso" establecido en la norma ISO 9000:2000[14]. Pueden identificarse tres partes: variables de entrada $x_{1}, x_{2}, \ldots x_{n}$; relación funcional entre las variables de entrada; el mensurando $y$ como variable de salida. El esquema se puede ver en la figura 1

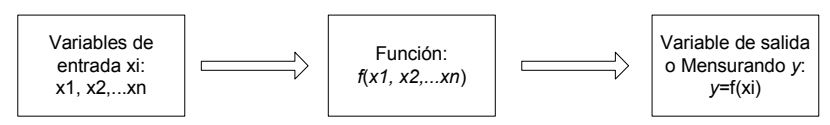

Figura 1. Proceso de medición de un mensurando.

Las variables de entrada son aleatorias que tienen una distribución de probabilidad conocida y pueden ser independientes o estar correlacionadas entre si[15].

El proceso de medición puede así representarse por un modelo matemático que relaciona el mensurando y las variables de entradas a través de la función $f$, tal a como se muestra en la ecuación (1); se asume ausencia de errores sistemáticos ${ }^{[15]}$. El segundo término representa la incertidumbre expandida del mensurando[6, 9]

Donde $U_{y}=k u_{y} ; u_{y}$ es la incertidumbre combinada; $k$

$$
y=f\left(x_{1}, x_{2}, \ldots x_{n}\right) \pm U_{y}
$$

es el factor de cobertura, que depende de los grados de libertad ${ }^{[6-9]}$ y de las diferentes distribuciones de probabilidad de las variables de entradas implicadas en la incertidumbre combinada. Para evaluar la incertidumbre combinada $u_{v}$ se aplica la ley de propagación de la incertidumbre ${ }^{[5,15]}$ a la función $f$. Esta ley se deduce aplicando la expansión de Taylor ${ }^{[11,15]}$, tomando $u y=\Delta y$ o diferencia entre la función $f(x i)$ y $f$ evaluada en los promedios de $x_{i}$. La expresión final que representa esta ley es la siguiente:

Si las variables $\left(x_{i}\right)$ son independientes, el segundo

$$
u_{(y)}^{2}=\sum\left(\frac{\partial f}{\partial x_{i}}\right)^{2} u_{x i}^{2}+2 \sum_{i=1}^{N-1} \sum_{j=i+1}^{N}\left(\frac{\partial f}{\partial x_{i}}\right)\left(\frac{\partial f}{\partial x_{j}}\right) u_{(x i, x j)}
$$

término denominado covarianza es despreciable. En los modelos lineales los coeficientes del modelo $\left(b_{i}\right)$ están correlacionados y debe tomarse en cuenta en el cálculo de la incertidumbre combinada.

Así por ejemplo, los coeficientes intercepto y pendiente del modelo de una línea recta $\left(b_{0}\right.$ y $\left.b_{1}\right)$ tienen una correlación negativa y debe calcularse el coeficiente de correlación $\left(r_{(b 0, b 1)}\right)$ para ser introducido en el término covarianza ${ }^{[6-9]}$.
Las derivadas parciales se denominan coeficientes de sensibilidad ${ }^{[6]}\left(c_{x i}\right) ; u_{x i}$ son las incertidumbres de las variables $x_{i}$

Si en el modelo matemático intervienen los parámetros de regresión de un modelo de calibración lineal (intercepto y pendiente) la ecuación (2) se puede expresar de la siguiente manera:

$$
u_{(y)}^{2}=\sum\left(c_{x i} u_{x i}\right)^{2}+2\left(c_{b 0}\right)\left(c_{b 1}\right) u_{(b 0)} u_{(b 1)} r_{(b 0, b 1)}
$$

Si se considera que no existen variables correlacionadas la incertidumbre $u_{y}$ se simplifica a la ecuación (4).

$$
u_{(y)}=\sqrt{\sum\left(c_{x i} u_{x i}\right)^{2}}
$$

Para evaluar la incertidumbre expandida, es necesario seleccionar el factor de cobertura $k$. Este factor puede tomar valores como los que se muestran en la tabla 1.

TABLA 1. Factor de cobertura, nivel de confianza y tipos de distribución de probabilidad

\begin{tabular}{|c|c|c|}
\hline$k$ & Nivel Confianza & $\begin{array}{c}\text { Tipo de } \\
\text { distribución }\end{array}$ \\
\hline 2 & $95.45 \%$ & Normal \\
\hline 1.96 & $95 . \%$ & Normal \\
\hline$t_{0.975, v}$ & $95 \%$ & Student, 2 colas \\
\hline
\end{tabular}

Generalmente, cuando se reporta la incertidumbre se selecciona $k=2$ para $95.45 \%$ de nivel de confianza ${ }^{[7]}$. Pero si se quiere efectuar una selección rigurosa de $k$ es necesario evaluar los grados de libertad efectivos $v_{\text {ef }}$ [6]; si éste tiene un valor menor de 30 , el factor $k$ sigue una distribución $t$ de Student. Los grados de libertad efectivos se calculan de acuerdo a la ecuación (5).

$$
v_{e f}=\frac{u_{u y}^{4}}{\sum \frac{c_{i}^{4} u_{x i}^{4}}{v_{i}}}
$$

$c_{x i}$ son los coeficientes de sensibilidad; $u_{x i}$ son las incertidumbres de la variable $x_{i}$ y $v_{i}$ son los grados de libertad para la incertidumbre de cada variable. La tabla 2 muestra los grados de libertad para cada tipo de distribución.

TABLA 2. Grados de libertad $v_{i}$ en función del tipo de distribución ${ }^{[6,}$ 20].

\begin{tabular}{|l|l|}
\hline \multicolumn{1}{|c|}{ Tipo de distribución } & $v_{i}$ \\
\hline Normal & 50 \\
\hline Rectangular & $\infty$ \\
\hline Triangular & $\infty$ \\
\hline$t$-Student (univariante) & $n_{i}-1$ \\
\hline$t$-Student (bivariante) & $n_{i}-2$ \\
\hline$t$-Student ( $p$ variables) & $n_{i}-p$ \\
\hline
\end{tabular}




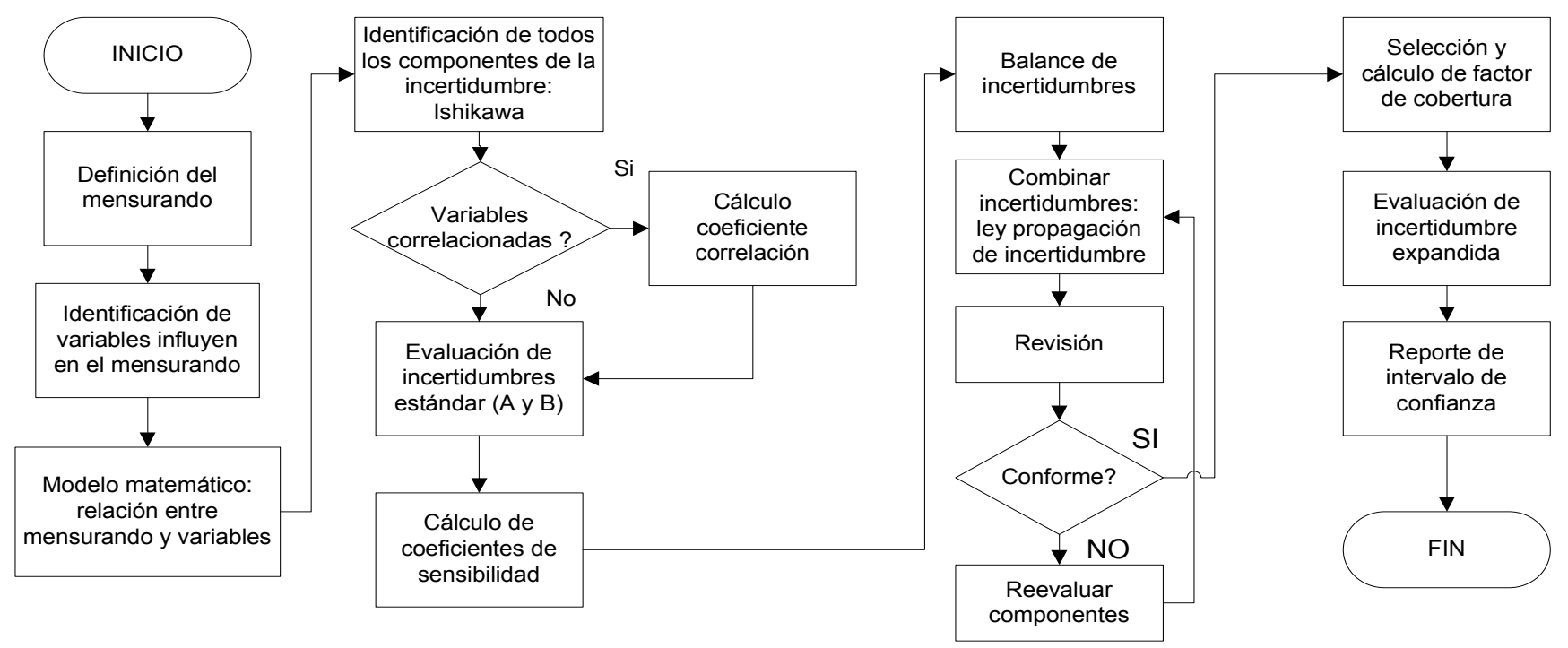

Figura 2: Diagrama de flujo representando el procedimiento para la estimación de la incertidumbre en un proceso de medición.

Conociendo el valor $k$ se puede estimar la incertidumbre expandida y el intervalo de confianza: $y \pm U y$.

\section{Procedimiento para la evaluación de la incertidumbre}

Existe un procedimiento para estimar la incertidumbre ${ }^{[6,9]}$, que se presenta en el diagrama de flujo de la figura 2. En primer lugar, se define el mensurando, se deriva el modelo matemático, se identifican las fuentes de incertidumbres (las de tipo A, obtenidas a partir de la desviación estándar de una serie de observaciones y las de tipo B, obtenidas a partir de las distribuciones normal, rectangular o triangular ${ }^{[6-9]}$, se cuantifican las incertidumbres, se calculan los coeficientes de sensibilidad, se hace un balance o presupuesto de las incertidumbres, se combinan las incertidumbres a través de la ley de la propagación de la incertidumbre, se revisa si no hay incongruencias para reevaluar los componentes de la incertidumbre, se selecciona el factor de cobertura, se evalúa la incertidumbre expandida y se reporta el intervalo de confianza con el número correcto de cifras significativas.

\section{Trazabilidad}

Todo instrumento de medición debe ser calibrado con patrones certificados trazables al Sistema Internacional de Unidades ${ }^{[4,16]}$. Para medir la longitud de un segmento es necesario que el instrumento de medición, por ejemplo una regla graduada en centímetros, esté calibrado con patrones certificados trazables a patrones nacionales y éstos a su vez trazables al SI.
El proceso de calibración, se debe llevar cabo bajo condiciones ambientales específicas (temperatura y humedad relativa, medidas también con instrumentos calibrados trazables). Si se asume que, los valores de las variables de entrada han sido obtenidos con instrumentos de medición calibrados con patrones trazables al SI, el mensurando $y$ de la ecuación (1) es también considerado trazable al Sistema Internacional [16].

Si las condiciones ambientales (temperatura) durante el proceso de medición del mensurando, son diferentes de las de calibrado del instrumento de medición, es necesario realizar las correcciones necesarias. Por ejemplo, si la regla graduada en centímetros es el instrumento de medición, la corrección a realizar debe tomar en cuenta el coeficiente de dilatación lineal, cuyo valor dependerá de la naturaleza de material del cual está hecho este instrumento, considerando la diferencia entre la temperatura de medición y la de calibrado.

\section{PARTE EXPERIMENTAL}

\section{Materiales}

- Computadora equipada con el programa de cálculo simbólico MAPLE $11^{[13]}$.

- Instrumento de medición: regla de madera impermeabilizada de $0.30 \mathrm{~m}$ de longitud, graduada en centímetros.

- Coeficiente de dilatación lineal del orden de $10^{-6} /$ ${ }^{\circ} \mathrm{C}^{[17]}$. 
- Objeto de medición: como el representado en la figura geométrica del anexo.

- Termómetro digital calibrado TESTO 925, con resolución $0.1^{\circ} \mathrm{C}$.

\section{PROCEDIMIENTO}

\section{Resolución del instrumento de medición}

La resolución de la regla graduada en centímetros fue evaluada por simple observación visual. El valor mínimo leído en la escala fue $0.05 \mathrm{~cm}$.

\section{Proceso de medición}

El objeto de medición y el instrumento de medición fueron proporcionados a un grupo de 10 estudiantes. Cada estudiante midió los segmentos de recta de la figura geométrica, que representa dos triángulos utilizando la misma regla y ubicando con precisión, el cero y el valor del extremo superior del segmento. Las mediciones fueron realizadas tratando de minimizar el error de paralaje y los resultados se registraron según la matriz de datos de la tabla 3. La variación de temperatura durante el proceso de medición fue de $2^{\circ} \mathrm{C}$.

TABLA 3. Diseño de los resultados de la medición en $\mathrm{cm}$ de los segmentos $a, b, .$. de la figura geométrica en anexo.

\begin{tabular}{|c|c|c|c|}
\hline No. & $a$ & $b$ & $\ldots \ldots$ \\
\hline 1 & & & \\
\hline 2 & & & \\
\hline. & & & \\
\hline. & & & \\
\hline. & & & \\
\hline 10 & & & \\
\hline
\end{tabular}

\section{RESULTADOS Y DISCUSIÓN}

Los resultados que a continuación se presentan, siguen el procedimiento descrito en el flujograma de la figura 1. Dado que, el coeficiente de dilatación lineal del instrumento de medición es muy pequeño, del orden de $10^{-6}$ por cada ${ }^{\circ} \mathrm{C}$, el efecto de la variación de temperatura, no influyó significativamente en las mediciones de los segmentos del triángulo. Como la regla está impermeabilizada, se asume que la humedad relativa no afecta la medición. Se admite que, la regla está calibrada y que el error sistemático, es mucho menor que el error aleatorio.

\section{Definición del mensurando}

El mensurando, es el área total de la figura geométrica del anexo, constituida por los dos triángulos rectángulos, y se designa simbólicamente como A.

\section{Modelo matemático}

El modelo para el área total del objeto de medición, es la suma de las áreas de los dos triángulos. La expresión matemática se presenta en la ecuación (6) admitiendo ausencia de errores sistemáticos:

$$
A=\frac{c}{2}(b+d)
$$

\section{Identificación de los componentes de la incertidumbre}

Los componentes de las incertidumbres para cada variable del modelo se muestran en el diagrama causa-efecto de la figura 3 . Cada variable tiene dos componentes: la incertidumbre debido a la resolución de la regla y la de la serie de observaciones de los 10 estudiantes.

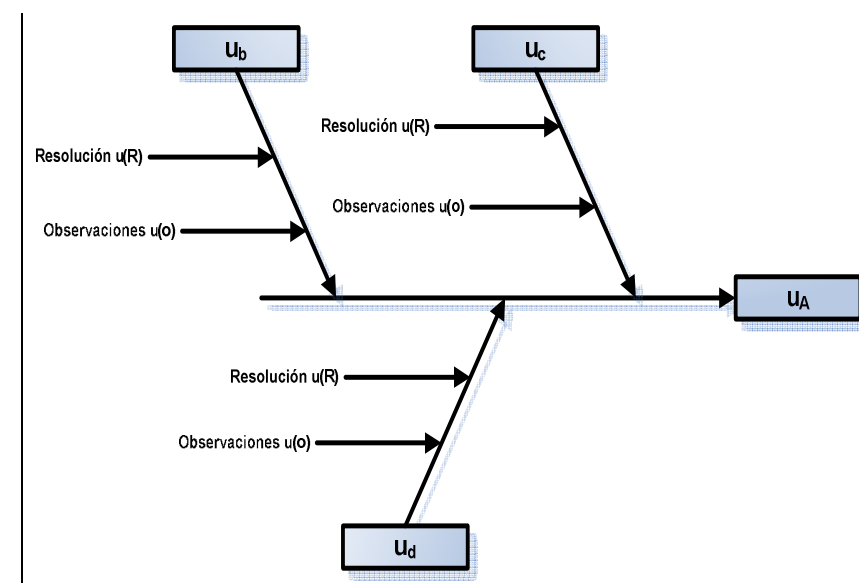

Figura 3. Diagrama causa-efecto para componentes de la incertidumbre en la medición del área de la figura geométrica del anexo.

\section{Cuantificación de los componentes de la incertidumbre}

\section{a) Incertidumbre en la resolución $u_{R}$}

La incertidumbre en la resolución (de tipo B) sigue una distribución rectangular ${ }^{[15]}$, la cual se expresa así:

$$
u_{R}=\frac{R}{2 \sqrt{3}}
$$

Sustituyendo $R=0.05 \mathrm{~cm}$ se tiene un valor de $u R=$ 0.0144 


\section{b) Incertidumbre en la serie de observaciones $u_{o}$}

La incertidumbre de una serie de observaciones (de tipo A) está dada por la desviación estándar de la media o clásicamente conocida como error estándar ${ }^{[18]}$. La ecuación (8) representa la fórmula para la incertidumbre de las observaciones.

$$
u_{o}=s_{\bar{x}}=\frac{s}{\sqrt{n}}
$$

Donde $s_{\bar{x}}$ es la desviación estándar de la media, $s$ es la desviación estándar de las observaciones y $n$ es el número de observaciones igual a 10 . Los resultados de las 10 observaciones del grupo de estudiantes se presentan en la tabla 4.

TABLA 4: Valores de los segmentos b, $c$, den cm, media, desviaciones estándares y grados de libertad $v_{i}$. Lecturas de 10 estudiantes.

\begin{tabular}{|c|c|c|c|}
\hline Estudiante & $b$ & $c$ & $d$ \\
\hline 1 & 8.3 & 7.85 & 4.55 \\
\hline 2 & 8.3 & 7.9 & 4.6 \\
\hline 3 & 8.3 & 7.9 & 4.6 \\
\hline 4 & 8.3 & 7.9 & 4.6 \\
\hline 5 & 8.25 & 7.9 & 4.6 \\
\hline 6 & 8.25 & 7.85 & 4.6 \\
\hline 7 & 8.3 & 7.85 & 4.55 \\
\hline 8 & 8.29 & 7.9 & 4.55 \\
\hline 9 & 8.3 & 7.9 & 4.55 \\
\hline 10 & 8.25 & 7.9 & 4.6 \\
\hline Media & 8.284 & 7.885 & 4.58 \\
\hline $\mathrm{s}$ & 0.02366432 & 0.02415229 & 0.02581989 \\
\hline $\mathrm{u}_{0}=\mathrm{s} / \sqrt{ } \mathrm{n}$ & 0.00748331 & 0.00763763 & 0.00816497 \\
\hline$v$ & 9 & 9 & 9 \\
\hline
\end{tabular}

c) Incertidumbre de las variables $b, c$ y $d\left(u_{x i}\right)$

La incertidumbre en las mediciones de los segmentos $b$, $c$ y $d$, se puede ver en la ecuación (9).

$$
u_{x i}=\sqrt{2 u_{R}^{2}+u_{o}^{2}}
$$

Generalizando se tiene la siguiente ecuación (10):

$$
u_{x i}=\frac{1}{6} \sqrt{6 R^{2}+\frac{36 s_{x i}}{n}}
$$

El coeficiente 2 del primer término en la raíz de la ecuación (9) representa la doble lectura en los extremos del segmento $x_{i}$, el cero leído en el extremo inferior y el valor de $x_{i}$ (en $\mathrm{cm}$ ) leído en el otro extremo.
El subíndice $x_{i}$ representa al segmento $b, c$ o $d$. R es la resolución de la regla graduada. $s_{x i}$ es la incertidumbre estándar del segmento leído. $\mathrm{n}$ es el número de observaciones. Sustituyendo los valores de $R=0.05 \mathrm{~cm}$ y los valores de $s_{x i}$ y $n$ de la tabla 4 en las ecuaciones (7), (8) y (10), se tienen los valores de incertidumbres en las mediciones $b, c$ y $d$ que se muestran en la tabla 5.

TABLA 5. Incertidumbres y grados de libertad para las variables $b, c$ $y d$.

\begin{tabular}{|l|c|c|c|}
\hline$x_{i}$ & $u_{R(v)}$ & $u_{o(v)}$ & $u_{x i(v i)}$ \\
\hline$b$ & $0.0144(\infty)$ & $0.00748331(9)$ & $0.021794(9)$ \\
\hline$c$ & $0.0144(\infty)$ & $0.00763763(9)$ & $0.021741(9)$ \\
\hline$d$ & $0.0144(\infty)$ & $0.00816497(9)$ & $0.021985(9)$ \\
\hline
\end{tabular}

Los grados de libertad $v_{i}$ se calculan combinando los grados de libertad de $R$ y o según la expresión representada en la ecuación 5 . De acuerdo a la tabla 2 , los grados de libertad para $u_{R}$ con una distribución triangula ${ }^{[15]}$ tienen un valor infinito y los grados de libertad para $u_{o}$ obtenidos para una serie de observaciones tienen una distribución $\mathrm{t}$ a un nivel de confianza de $95 \%$ y n-1 grados de libertad.

\section{Balance de las incertidumbres}

El balance de las incertidumbres establece las incertidumbres de las variables del modelo $u_{x i}$, los coeficientes de sensibilidad $c_{x i}$ y el índice de contribución de cada variable $x_{i}$ en la incertidumbre de la función del modelo o el mensurando buscado, que se representa como porcentaje (Ind\%). Los coeficientes de sensibilidad son la derivada parcial de la función $A$ con respecto a cada variable $x_{i}$.

\section{Cálculo de los coeficientes de sensibilidad $c_{x i}$ :}

$$
c_{x i}=\frac{\partial A}{\partial x_{i}}
$$

Las derivadas parciales se pueden calcular fácilmente, dado que la función A de la ecuación (6) es simple. Cuando las funciones son complejas el cálculo simbólico Maple 11 simplifica el tiempo de cálculo. Además la precisión es ilimitada y no utiliza redondeos. Aplicando Maple para este ejemplo se tienen los siguientes resultados formales:

Se escribe la función A:

$$
>A:=\frac{c}{2} *(b+d)
$$

$$
\frac{c}{2}(b+d)
$$


Se deriva la función $A$ con respecto a los segmentos $c, b$ y $d$, utilizando las expresiones preestablecidas de derivadas parciales:

$$
\begin{aligned}
& >c 1:=\frac{\partial}{\partial c} A ; \\
& >c 2:=\frac{\partial}{\partial b} A \\
& >c 3:=\frac{\partial}{\partial d} A ;
\end{aligned}
$$

donde $\mathrm{c}_{1}, \mathrm{c}_{2} \mathrm{y} \mathrm{c}_{3}$ indican los coeficientes de sensibilidad para $c, b$ y $d$. Asignando los valores promedios $c, b$ y $d$ a partir de la tabla 4 , se tienen los resultados numéricos de $c_{x i}$.

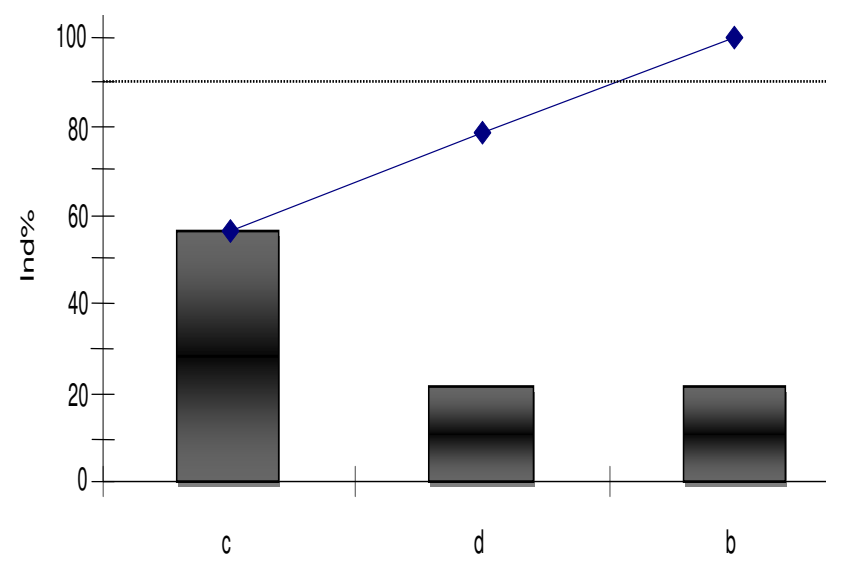

Figura 4. Diagrama de Pareto indicando la contribucion de las incertidumbres en la medición del área total del triangulo.
La tabla 6 muestra el balance de las incertidumbres, donde se pueden ver las fuentes de incertidumbres $(b$, $c$ y $d$ ), el valor de cada variable $x_{i}$, las incertidumbres y los coeficientes de sensibilidad correspondientes, los valores absolutos y los cuadrados del producto de los coeficientes por las incertidumbres y por último, el índice de contribución de la incertidumbre de cada variable en la incertidumbre del mensurando.

La contribución de cada variable a la incertidumbre del área total de la figura geométrica se evalúa con el índice de contribución expresado en porcentaje según la ecuación siguiente:

$$
\text { Ind } \%=\frac{\left(c_{x i} u_{x i}\right)^{2}}{\sum\left(c_{x i} u_{x i}\right)^{2}}(100
$$

Si trazamos el diagrama de Pareto ${ }^{[19]}$ se tiene la figura 4 , donde se puede ver que la variable que más contribuye a la incertidumbre es la medición en el segmento c. No obstante, las tres mediciones de los segmentos contribuyen significativamente a la incertidumbre en el mensurando $\mathrm{A}$, admintiendo como límite el $90 \%$ de la contribución acumulativa.

\section{Estimación de la incertidumbre combinada $u_{A}$}

La incertidumbre de $\mathrm{A}\left(u_{A}\right)$ es la combinación de todas las incertidumbres que influyen en la medición del mensurando $A$. Se asume que las variables de la función son independientes y se aplica la ecuación (4). La incertidumbre del mensurando $A$ es la raíz cuadrada de la suma de los productos $\left(c_{x i} u_{x i}\right)^{2}$ en la columna 6 de la tabla 6 , cuyo valor es:

$$
u_{A}=0.1858
$$

\begin{tabular}{|c|c|c|c|c|c|c|}
\hline $\begin{array}{c}\text { Fuente de } \\
\text { incertidumbre } \\
x_{i}\end{array}$ & $\begin{array}{c}\text { Valor de } \\
\quad x i\end{array}$ & $\mu_{x i}$ & $c_{x i}$ & $/ c_{x i} m_{x i} /$ & $\left(c_{x i} \mu_{x i}\right)^{2}$ & Ind $\%$ \\
\hline$b$ & 8.284 & 0.021794 & 3.9425 & 0.08571 & 0.0073468 & 21.29 \\
\hline$c$ & 7.885 & 0.021741 & 6.432 & 0.14018 & 0.0196651 & 56.94 \\
\hline$d$ & 4.580 & 0.021985 & 3.9425 & 0.086675 & 0.0075126 & 21.77 \\
\hline \multicolumn{5}{|r|}{ Suma= } & 0.0345104 & 100 \\
\hline
\end{tabular}

\section{Selección del factor de cobertura $k$}

Si se observa la tabla 5 , la distribución $t$ es la predominante en la estimación de $u_{x i}$. Por lo tanto, si se aplica la ecuación (5) y tomando los datos de la tabla 6, se obtienen los grados de libertad efectivos:

TABLA 6. Balance de las incertidumbres en la medición del área de un triángulo como objeto de medición. 


$$
\begin{gathered}
v_{e f}=\frac{(0.1858)^{4}}{\frac{(3.9425)^{4}(0.021794)^{4}}{9}+\frac{(6.432)^{4}(0.021741)^{4}}{9}+\frac{(3.9425)^{4}(0.021985)^{4}}{9}} \\
v_{\text {ef }}=22
\end{gathered}
$$

El valor de $k$ es igual a $t$ al $95 \%$ de nivel de confianza a dos colas y 22 grados de libertad ${ }^{[6,18]}$.

$$
k=t_{0.975,22}=2.074
$$

\section{Evaluación de la incertidumbre expandida}

La incertidumbre expandida, se calcula multiplicando el factor $k$ por la incertidumbre combinada $U_{A}$. El cálculo se muestra a continuación:

$$
U_{A}=k u_{A}=(2.074)(0.1858)=0.38535 \mathrm{~cm}^{2}
$$

\section{Estimación del intervalo de confianza del área del triángulo}

El intervalo de confianza del área del triángulo con una probabilidad del $95 \%$ y tomando en cuenta el número de cifras significativas correcto, se calcula de la siguiente manera:

$$
\begin{gathered}
y=A \pm U_{A}=\frac{7.885}{2}(8.284+4.58) \pm 0.38535 \\
y=(50.72 \pm 0.39) \mathrm{cm}^{2}
\end{gathered}
$$

La precisión de la medición puede estimarse evaluando la incertidumbre expandida relativa ${ }^{[11]}$ (UER) expresada en porcentaje, la cual resulta de dividir la incertidumbre expandida entre el valor del mensurando o área total multiplicada por 100 . El valor calculado de esta manera es de $0.77 \%$, lo que indica una buena precisión en la medición si tomamos como referencia $1 \%$ como máximo.

\section{CONCLUSIONES}

Siguiendo la guía para la estimación de la incertidumbre establecida en GUM ISO 1995, se aplicó el procedimiento para evaluar la incertidumbre en la medición del área total de un triángulo, realizando las mediciones un grupo de 10 estudiantes de maestría en química y gestión de la calidad de la Facultad de Ciencias y Tecnología de la UNAN-León, Nicaragua.

En el cálculo de los coeficientes de sensibilidad se utilizó el programa de cálculo simbólico MAPLE 11, como una aplicación de las derivadas parciales de la función área con respecto a cada una de las variables o segmentos que constituyen el triángulo. Para evaluar la incertidumbre combinada en el proceso de medición, se aplicó la ecuación simplificada de la ley de propagación de la incertidumbre, considerando que las variables son independientes.

El intervalo de confianza del área del triángulo, se evalúo la incertidumbre expandida utilizando el factor de cobertura $k$ como el valor de $t$ al $95 \%$ de nivel de confianza y 22 grados de libertad efectivos. El valor encontrado del intervalo de confianza fue de $(50.72 \pm 0.39) \mathrm{cm}^{2}$.

\section{RECONOCIMIENTO}

Los autores agradecen a Gustavo Montiel, Director del Laboratorio Nacional de Metrología de Nicaragua (LANAMET) y a Miguel Carrero Gálvez, Consultor de Asesores de Calidad y Medioambiente (CACM-España), por sus valiosos comentarios y sugerencias al presente trabajo.

\section{BIBLIOGRAFÍA}

1. LEY No225 (1996). "Ley sobre Metrología", La Gaceta No. 135, República de Nicaragua.

2. ISO/IEC 17000 (ES) (2004). "Evaluación de la conformidad - Vocabulario y Principios Generales", Ginebra.

3. ISO/IEC 17011(ES) (2004). "Evaluación de la conformidad - Requisitos generales para los organismos de acreditación que realizan la acreditación de organismos de evaluación de la conformidad", Norma Internacional, Ginebra

4. ISO/IEC 17025(ES) (2005). "Requisitos Generales para la Competencia de los Laboratorios de Ensayo y de Calibración", Norma Internacional, Ginebra, Segunda Edición.

5. ILAC-G8 (1966). "Guidelines on Assessment and Reporting of compliance with specification".

6. BIPM, IEC, IFCC, IUPAC, OIML (1995). Guide for to the Expression of Uncertainty in Measurement (GUM), ISO, Ginebra.

7. NIST (1993). "Guidelines for evaluation and expressing the uncertainty of NIST measurement results", Gaithersburg, USA. 
8. SCHMID, W.A. y LAZOS, R.J. (2000), "Guía para estimar la incertidumbre de la medición", CENAM, México.

9. EURACHERM/CITAC GUIDE, (2000), Quantifying Uncertainty in Analytical Chemistry, 2da edición, UK.

10. DELGADO M, VANEGAS M y DELGADO G. (2007), "Metrología Química I: Calibración de un pH-metro y control de calidad", UNIVERSITAS UNAN-León, 1, 1420.

11. DELGADO M, VANEGAS M y DELGADO G. (2007) "Metrología Química II: Estimación de la incertidumbre en la medición de $\mathrm{pH}$ en aguas", UNIVERSITAS UNANLéon, 2, 19-26.

12. YATES P. C. (2001), "A simple for illustrating uncertainty analysis", Journal of Chemical Education, 78, 770-771.

13. a) CHAR B. W. (1992), Maple V, Springer-Verlag, New York, pag. 41; b) Maple 11, software.

14. ISO 9000:2000 (ES), (2000), Sistemas de Gestión de la Calidad. Fundamentos y Vocabulario.

15. KIMOTHI, S.K., (2002), The Uncertainty of Measurements, ASQ Quality Press, Wisconsin , pag. 205.

16. EURACHEM/CITAC GUIDE, (2003), Treceability in chemical measurement, Guía de Referencia, 1ra. Edición, UK.

17. DEAN, J. A. (1979), Lange's Hanbook of Chemistry, McGraw Hill, pag. 10-119.

18. NEULLY, M. (1998), Modélisation et estimation des erreurs de mesure, Technique \& Documentation, $2^{\mathrm{a}}$ edición, pag. 296.

19. WODSWORTH, H. M., STEPHENS, K. y GODFREY, A. B. (2005), Métodos de Control de Calidad, México, Compañía Editorial Continental, 1ra. Edición, p. 362.

20. CHRZANOW (2002). "For evaluation of uncertainty in mesasurements", Joint IMEKO TC-1 \& XXXIV Conference 2002, 11- 12 septiembre, Warsaw.

\section{ANEXO}

Figura geométrica como objeto de medición. La escala de la figura original utilizada durante el experimento, puede variar al pasar de MS Word a la configuración del editor de texto utilizado en la revista científica.

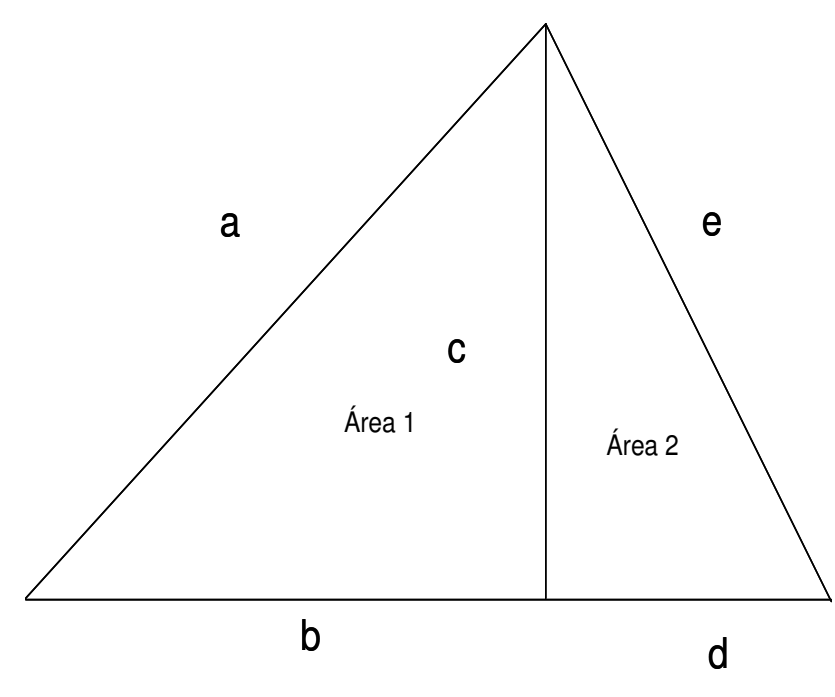

